\title{
Cascade Reliability of Stress-Strength system when Strength follows mixed Exponential distribution
}

\author{
${ }^{1}$ T.Sumathi Uma Maheswari, ${ }^{2}$ N.Swathi \\ ${ }^{1,2}$ Department of Mathematics, Kakatiya University, Warangal- 506009, Andhra Pradesh
}

\begin{abstract}
Cascade reliability model is a special type of Stress- Strength model. The $n$-Cascade system is a hierarchical standby redundancy system, where the standby component taking the place of failed component with decreased value of stress and independently distributed strength.

In assessing system reliability it is first necessary to define and categorize different modes of system failures. The individual distributions that are combined to form the mixture distribution are called mixer components. In this paper it has been discussed that the reliability of $n$-cascade system when strength follows mixed exponential distribution and stress follows exponential distribution.
\end{abstract}

\section{Introduction}

An $n$ - cascade system is defined as a special type of standby system with $n$ components by Sriwastav et al.,[2]. Cascade redundancy is defined as a hierarchical standby redundancy where a standby component takes the place of a failed component with a changed stress. This changed stress is $k$ times the preceding stress. $k$ is the attenuation factor.

Sriwastav and Pandit[2] derived the expressions for reliability of an $n$-cascade system when stress and strength follow exponential distribution. They computed reliability values for a 2-cascade system with gamma and normal stress and strength distributions. Raghava Char et al [3] studied the reliability of a cascade system with normal stress and strength distribution. T.S.Uma Maheswari et al [4] studied the reliability comparison of $\mathrm{n}$-cascade system with addition of $\mathrm{n}$-strengths system when stress and strength follow exponential distribution. They concluded that when the attenuation factor is less than 0.5 the cascade model has more reliability and at each attack if the stress decreases more than cascade model is more reliable. Uma Maheswari et al [5] derived the reliability of n-cascade system with normal stress and exponential strength. They concluded that the marginal reliability rate increases for higher values of strength parameter. Rekha and Shyam Sunder [6] studied the reliability of a cascade system with exponential strength and gamma stress. They showed that for higher parametric values and lower attenuation factors a high degree of reliability could be attained. Rekha and Chenchu Raju [7] studied the stress attenuated cascade reliability when both stress and strength follow rayleigh distribution. They concluded that for lower attenuation factors a high degree of reliability could be obtained.

\section{Statistical Model}

If $X$ denotes the strength of the component and $Y$ is the stress imposed on it, then the reliability of the component is given by[1],

$$
R=P(X>Y)=\int_{0}^{\infty}\left\{\int_{0}^{x} g(y) d y\right\} f(x) d x
$$

Let $X_{1}, X_{2}, X_{3}, \ldots, X_{n}$ be the strengths of the components $C_{1}, C_{2}, C_{3}, \ldots, C_{n}$ as arranged in order of activation respectively. All the $X_{i}$ 's are independent and identically distributed random variables with probability density functions $f_{i}\left(x_{i}\right) ; i=1,2, \ldots, n$. Also let $Y_{1}$ be the stress on the first component which is also randomly distributed with the density function $g\left(y_{1}\right)$.

If $Y_{1}<X_{1}$, the first component which is also randomly distributed stress varies with the density function $g\left(y_{1}\right)$. If $Y_{1}<X_{1}$, the first component $C_{1}$ works and hence the system survives. $Y_{1} \geq X_{1}$ leads to the failure of $C_{1}$; thus the second component in line viz., $C_{2}$, takes its place and has a strength $X_{2}$. However, the stress $Y_{2}$ on $C_{2}$ will normally be different from $Y_{1}$. Let $Y_{2}=K_{2}^{*} Y_{1}$, where $K_{2}^{*}$ is the cumulative attenuation factor on the second component and $K_{2}^{*}=K_{1} K_{2}$ where by definition $K_{1}=1$.Although the system has suffered the loss of one component, it survives if $Y_{2}<X_{2}$ and so on . In general, if the $(i-1)^{\text {th }}$ component $C_{i-1}$ fails then the $i^{\text {th }}$ component $C_{i}$, with the strength $X_{i}$, gets activated and will be subjected to the stress.

$$
\begin{array}{cc} 
& Y_{i}=K_{i} Y_{i-1}=K_{i}^{*} Y_{1} \\
\text { where } & K_{i}^{*}=K_{1} K_{2} \ldots \ldots K_{i}
\end{array}
$$

represents the cumulative attenuation factor on the $i^{\text {th }}$ component $C_{i}$. 
The system could survive with a loss of the first $(n-1)$ components if and only if $X_{i} \leq Y_{i} ; i=1,2,3, \ldots \ldots, n-1$ and $X_{n}>Y_{n}$. The system totally fails if all the components fail when $X_{i} \leq$ $Y_{i} ; i=1,2, \ldots \ldots n$.

The probability $R(n)$ of the system to survive with the first $(n-1)$ components failed and the $n^{\text {th }}$ component active is

$$
R(n)=P\left[\left\{\bigcap_{i=1}^{n-1}\left(X_{i} \leq Y_{i}\right)\right\} \cap\left(X_{n}>Y_{n}\right)\right]
$$

$R(2), R(3)$,

$R(n)$ are the increments in reliability due to the addition of $2^{\text {nd }}, 3^{\text {rd }}$ $n^{\text {th }}$ components respectively.

Then

$R(n)=P\left[X_{1} \leq K_{1}^{*} Y_{1}, X_{2} \leq K_{2}^{*} Y_{1}, \ldots \ldots \ldots, Y_{1}, X_{n-1} \leq K_{n-1}^{*} Y_{1}, X_{n}>K_{n}^{*} Y_{1}\right]$

we can obviously associate the $n^{\text {th }}$ component attenuation factor with $Y_{1}$.

The distribution of $Y_{1}$ is the specified if the distribution of $Y_{2}, Y_{3} \ldots \ldots$ are fixed. Hence it is necessary to specify the distribution of $Y_{1}$. Let $g\left(y_{1}\right)$ and $f_{i}\left(x_{i}\right)$ be the probability density function of $Y_{1}$ and $X_{i}(i=$ $1,2, \ldots, n$ respectively.

The equation (4) can now be written as

$$
\begin{aligned}
R(n)=\int_{0}^{\infty}\left[\int_{0}^{K_{1}^{*} y_{1}} f_{1}\left(x_{1}\right) d x_{1} \times \int_{0}^{K_{2}^{*} y_{1}} f_{2}\left(x_{2}\right) d x_{2} \times \ldots \ldots \int_{0}^{K_{n-1}^{*} y_{1}} f_{n-1}\left(x_{n-1}\right) d x_{n-1}\right. \\
\left.\quad \times \int_{K_{n}^{*} y_{1}}^{\infty} f_{n}\left(x_{n}\right) d x_{n}\right] g\left(y_{1}\right) d y_{1}
\end{aligned}
$$

(or)

$$
\begin{gathered}
\int_{0}^{\infty}\left[F_{1}\left(K_{1}^{*} y_{1}\right) F_{2}\left(K_{2}^{*} y_{1}\right) \ldots \ldots \ldots F_{n-1}\left(K_{n-1}^{*} y_{1}\right) \bar{F}_{n}\left(K_{n}^{*} y_{1}\right)\right] g\left(y_{1}\right) d y_{1} \\
\text { where } F_{i}\left(K_{i}^{*} y_{1}\right)=\int_{0}^{K_{i}^{*} y_{1}} f_{i}\left(x_{i}\right) d x_{i} \quad \text { and } \\
\bar{F}_{i}\left(K_{i}^{*} y_{1}\right)=1-F_{i}\left(K_{i}^{*} y_{1}\right)
\end{gathered}
$$

Let $X_{1}$ and $X_{2}$ be the two strengths additively acted on a system and $X$ be the strength composed on it with pdf's

$$
\begin{array}{cc}
g\left(y_{i}\right)=\mu e^{-\mu y_{i}} & i=1,2 \ldots . \mathrm{n} \\
f(x)=\lambda e^{-\lambda x} & \lambda \geq 0
\end{array}
$$

Let $X_{1}$ and $X_{2}$ are additive in the ratio $p_{1}, p_{2}$ acted on a single stress system then the combined strength $X=$ $p_{1} X_{1}+p_{2} X_{2}$ and $p_{1}+p_{2}=1$

Its density function is

Transformation

$$
f(x)=\int_{0}^{y} f_{1}\left(p_{1} x_{1}\right) f_{2}\left(p_{2} x_{2}\right) d x_{1} d x_{2}
$$

$$
\begin{gathered}
U=p_{1} X_{1}+p_{2} X_{2} \\
V=X_{2} \quad X_{1}=\frac{U-p_{2} V}{p_{1}} \\
J=\left|\begin{array}{cc}
\partial X_{1} & \partial X_{2} \\
\partial U & \partial V
\end{array}\right|=\left|\begin{array}{cc}
\frac{\partial X_{1}}{\partial u} & \frac{\partial X_{2}}{\partial u} \\
\frac{\partial X_{1}}{\partial v} & \frac{\partial X_{2}}{\partial v}
\end{array}\right|=\left|\begin{array}{cc}
\frac{1}{p_{1}} & 0 \\
\frac{-p_{2}}{p_{1}} & 1
\end{array}\right|=\frac{1}{p_{1}} \\
f(u, v)=\lambda^{2} e^{-\lambda x_{1}-\lambda x_{2}} \\
=\lambda^{2} e^{-\lambda\left(\frac{u-p_{2} v}{p_{1}}\right)-\lambda v} \lambda^{2} e^{-\frac{\lambda u}{p_{1}}} e^{\left(\frac{\lambda p_{2}}{p_{1}}-\lambda\right) v}
\end{gathered}
$$




$$
\begin{gathered}
f(u)=\lambda^{2} e^{-\frac{\lambda u}{p_{1}}} \int_{0}^{u} e^{\left(\frac{\lambda p_{2}}{p_{1}} \lambda\right) v} d v=\frac{\lambda^{2} e^{-\frac{\lambda u}{p_{1}}}}{\lambda p_{2}-\lambda p_{1}}\left[e^{-\left(\frac{\lambda p_{2}-\lambda p_{1}}{p_{1}}\right) u}-1\right] \\
=\frac{\lambda^{2}}{\lambda p_{2}-\lambda p_{1}}\left[e^{-\lambda\left(\frac{1-p_{2}}{p_{1}}\right) u}-e^{-\frac{\lambda u}{p_{1}}}\right] \\
f(u)=\frac{\lambda^{2}}{\lambda p_{2}-\lambda p_{1}}\left[e^{-2 \lambda u}-e^{-\frac{\lambda u}{p_{1}}}\right] \\
f(x)=\frac{\lambda}{p_{2}-p_{1}}\left[e^{-2 \lambda x}-e^{-\frac{\lambda x}{p_{1}}}\right]
\end{gathered}
$$

Then $\mathrm{n}$ - cascade Reliability

Then 1-Cascade is

$$
R(n)=P\left[\bigcap_{i=1}^{n-1}\left(X_{i}<Y_{i}\right) \cap\left(X_{n}>Y_{n}\right)\right]
$$

$$
\begin{gathered}
R(1)=P\left(X_{1}>Y_{1}\right) \\
R(1)=\int_{x=0}^{\infty}\left(\int_{y=0}^{x} g(y) d y\right) f(x) d x=\int_{x=0}^{\infty}(G(x)) f(x) d x
\end{gathered}
$$

Where $G(x)=\int_{y=0}^{x} g(y) d y=\int_{0}^{x} \mu e^{-\mu y} d y=\left(1-e^{-\mu x}\right)$

$$
\begin{aligned}
& R(1)=\int_{x=0}^{\infty}\left(1-e^{-\mu x}\right)\left(\frac{\lambda}{p_{2}-p_{1}}\right)\left(e^{-2 \lambda x}-e^{\frac{-\lambda x}{p_{1}}}\right) d x \\
= & \frac{\lambda}{p_{2}-p_{1}} \int_{0}^{\infty}\left(e^{-(\mu+2 \lambda) x}-e^{-\left(\frac{\lambda}{p_{1}}+\mu\right) x}+e^{-2 \lambda x}-e^{\frac{\lambda}{p_{1}} x}\right) d x \\
= & \frac{\lambda}{p_{2}-p_{1}}\left[\frac{1}{2 \lambda}-\frac{p_{1}}{\lambda}-\frac{1}{\mu+2 \lambda}+\frac{p_{1}}{p_{1} \mu+\lambda}\right]
\end{aligned}
$$

2-Cascade system

$$
R(2)=P\left(X_{1}<Y_{1}, X_{2}>Y_{2}\right)=P\left(X_{1}<Y, X_{2}>K_{1}^{*} Y\right)
$$

$$
\begin{gathered}
=\int_{x=0}^{\infty}(1-G(x))\left(G\left(K^{*}{ }_{1} X\right)\right) f(x) d x \\
=\int_{x=0}^{\infty} e^{-\mu x}\left(1-e^{-\mu x K_{1}^{*}}\right) \frac{\lambda}{p_{2}-p_{1}}\left(e^{-2 \lambda x}-e^{\frac{-\lambda x}{p_{1}}}\right) d x \\
=\frac{\lambda}{p_{2}-p_{1}} \int_{x=0}^{\infty}\left(e^{-\mu x}-e^{-\mu x\left(K_{1}^{*}+1\right)}\right)\left(e^{-2 \lambda x}-e^{\frac{-\lambda x}{p_{1}}}\right) d x \\
=\frac{\lambda}{p_{2}-p_{1}} \int_{0}^{\infty}\left(e^{-(\mu+2 \lambda) x}-e^{-\left(\mu+\frac{\lambda}{p_{1}}\right) x}-e^{-\left(\mu\left(1+K_{1}^{*}\right)+2 \lambda\right) x}+e^{-\left(\mu\left(1+K_{1}^{*}\right)+\frac{\lambda}{p_{1}}\right) x}\right) d x \\
\frac{\lambda}{p_{2}-p_{1}}\left[\frac{1}{\mu+2 \lambda}-\frac{p_{1}}{\mu p_{1}+\lambda}-\frac{1}{\mu\left(1+K_{1}^{*}\right)+2 \lambda}+\frac{p_{1}}{p_{1} \mu\left(1+K_{1}^{*}\right)+\lambda}\right] \\
=\frac{\lambda}{p_{2}-p_{1}} \int_{x=0}^{\infty}(1-G(X))\left(1-G\left(K_{1}^{*} X\right)\right)\left(G\left(K_{2}^{*} X\right)\right) f(x) d x \\
R(3)=P\left(X_{1}<Y_{1}, X_{2}<Y_{2}, X_{3}>Y_{3}\right) \\
-K_{1}^{*} x \mu \\
\int_{x}\left(1-e^{-K_{2}^{*} x \mu}\right)\left(e^{-2 \lambda x}-e^{\frac{-\lambda x}{p_{1}}}\right) d x
\end{gathered}
$$

3-Cascade system 


$$
\begin{gathered}
=\frac{\lambda}{p_{2}-p_{1}} \int_{y=0}^{\infty}\left(e^{-\left(\mu\left(1+K_{1}^{*}\right) x\right.}\right)\left(1-e^{-K_{2}^{*} x \mu}\right)\left(e^{-2 \lambda x}-e^{\frac{-\lambda x}{p_{1}}}\right) d x \\
=\frac{\lambda}{p_{2}-p_{1}} \int_{y=0}^{\infty}\left(e^{-\left(\mu\left(1+K_{1}^{*}\right) x\right.}-e^{-\left(K_{1}^{*}+K_{2}^{*}+1\right) x \mu}\right)\left(e^{-2 \lambda x}-e^{\frac{-\lambda x}{p_{1}}}\right) d x \\
=\frac{\lambda}{p_{2}-p_{1}} \int_{y=0}^{\infty} e^{-\left(\mu\left(1+K_{1}^{*}\right)+2 \lambda\right) x}-e^{-\left(\mu\left(1+K_{1}^{*}\right)+\frac{\lambda}{p_{1}}\right) x}-e^{-\left(K_{1}^{*}+K_{2}^{*}+1+2 \lambda\right) x \mu}+e^{-\left(K_{1}^{*}+K_{2}^{*}+1+\frac{\lambda}{p_{1}}\right) x \mu} d x \\
R(3)=\frac{\lambda}{p_{2}-p_{1}}\left[\frac{1}{\mu\left(1+K_{1}^{*}\right)+2 \lambda}-\frac{p_{1}}{p_{1} \mu\left(1+K_{1}^{*}\right)+\lambda}-\frac{1}{\mu\left(1+K_{1}^{*}+K_{2}^{*}\right)+2 \lambda}\right. \\
\left.+\frac{p_{1} \mu(13)}{p_{1} \mu\left(K_{1}^{*}+K_{2}^{*}+1\right)+\lambda}\right]
\end{gathered}
$$

4- Cascade system

$$
\begin{aligned}
R(4)=\frac{\lambda}{p_{2}-p_{1}} & {\left[\frac{1}{\left(1+K_{1}^{*}+K_{2}^{*}\right) \mu+2 \lambda}-\frac{p_{1}}{p_{1} \mu\left(1+K_{1}^{*}+K_{2}^{*}\right)+\lambda}-\frac{1}{\mu\left(1+K_{1}^{*}+K_{2}^{*}+K_{3}^{*}\right)+2 \lambda}\right.} \\
& \left.+\frac{p_{1}}{p_{1} \mu\left(1+K_{1}^{*}+K_{2}^{*}+K_{3}^{*}\right)+\lambda}\right]
\end{aligned}
$$

n- Cascade system

$$
\begin{aligned}
& R(n)=\frac{\lambda}{p_{2}-p_{1}}[ \frac{1}{\left(1+K_{1}^{*}+K_{2}^{*}+\cdots . . K_{n-2}^{*}\right) \mu+2 \lambda}-\frac{p_{1}}{p_{1} \mu\left(1+K_{1}^{*}+K_{2}^{*}+\cdots K_{n-2}^{*}\right)+\lambda} \\
&-\frac{1}{\mu\left(1+K_{1}^{*}+K_{2}^{*}+K_{3}^{*}+\cdots+K_{n-1}^{*}\right)+2 \lambda} \\
&\left.+\frac{p_{1}}{p_{1} \mu\left(1+K_{1}^{*}+K_{2}^{*}+K_{3}^{*}+\cdots . . K_{n-1}^{*}\right)+\lambda}\right] \\
& R(n)=\frac{\lambda}{p_{2}-p_{1}}\left[\frac{1}{\mu\left(\sum_{i=0}^{n-2} K_{i}^{*}\right)+2 \lambda}-\frac{p_{1}}{p_{1} \mu\left(\sum_{i=0}^{n-2} K_{i}^{*}\right)+\lambda}-\frac{1}{\mu\left(\sum_{i=0}^{n-1} K_{i}^{*}\right)+2 \lambda}+\frac{p_{1}}{p_{1} \mu\left(\sum_{i=0}^{n-1} K_{i}^{*}\right)+\lambda}\right]
\end{aligned}
$$

\section{Reliability Computations}

Table 1

\begin{tabular}{|c|c|c|c|c|c|c|c|c|c|}
\hline$\lambda$ & $\mathrm{p} 1$ & $\mathrm{p} 2$ & $\mu$ & $\mathrm{k} 1 *$ & $\mathrm{k} 2^{*}$ & $\mathrm{R}(1)$ & $\mathrm{R}(2)$ & $\mathrm{R}(3)$ & $\mathrm{R}(4)$ \\
\hline 0.1 & 0.1 & 0.9 & 5 & 2 & 3 & 0.455128 & 0.02794 & 0.0129 & 0.0223 \\
\hline 0.2 & 0.2 & 0.8 & 5 & 2 & 3 & 0.382716 & 0.05361 & 0.0676 & 0.0752 \\
\hline 0.3 & 0.3 & 0.7 & 5 & 2 & 3 & 0.241071 & 0.07727 & 0.2266 & 0.3522 \\
\hline 0.4 & 0.4 & 0.6 & 5 & 2 & 3 & 0.17816 & 0.09912 & 0.4033 & 0.5153 \\
\hline
\end{tabular}

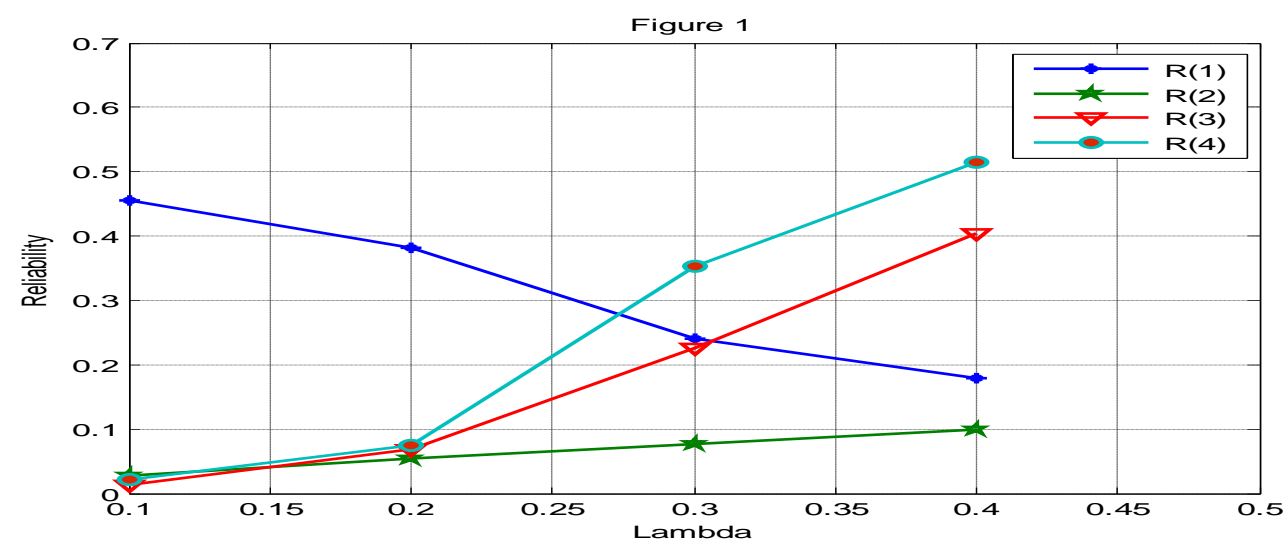

Table 2 


\begin{tabular}{|c|c|c|c|c|c|c|c|c|r|}
\hline$\lambda$ & $\mathrm{p} 1$ & $\mathrm{p} 2$ & $\mu$ & $\mathrm{k} 1 *$ & $\mathrm{k} 2 *$ & $\mathrm{R}(1)$ & $\mathrm{R}(2)$ & $\mathrm{R}(3)$ & $\mathrm{R}(4)$ \\
\hline 0.3 & 0.1 & 0.9 & 1 & 2 & 3 & 0.1718 & 0.0989 & 0.1598 & 0.2835 \\
\hline 0.3 & 0.2 & 0.8 & 2 & 2 & 3 & 0.1648 & 0.0403 & 0.1231 & 0.2231 \\
\hline 0.3 & 0.3 & 0.7 & 3 & 2 & 3 & 0.1041 & 0.0177 & 0.0199 & 0.0352 \\
\hline 0.3 & 0.4 & 0.6 & 4 & 2 & 3 & 0.0188 & 0.0087 & 0.0095 & 0.0162 \\
\hline
\end{tabular}

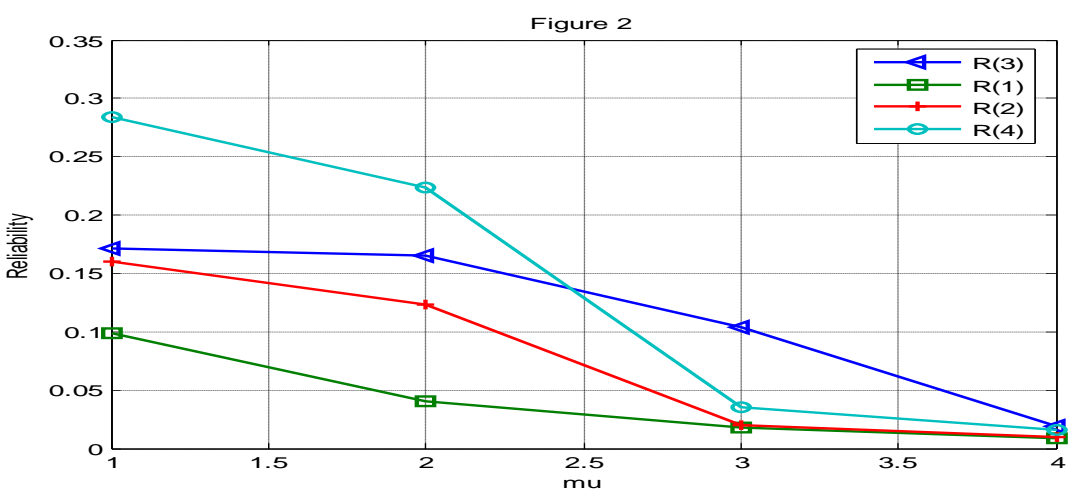

\section{Conclusion}

The reliability of $\mathrm{n}$ - cascade system when stress follows mixed exponential distribution and strength follows exponential distribution. In this paper we find out formula for the Reliability of $\mathrm{n}$ - cascade system.

\section{References}

[1] Kapur, K.C and L.R.Lamberson,( 1977). Reliability in Engineering Design ,Jhon Wiley and Sons, Inc., New York.

[2] S.N.Narahari Pandit and G.L.Sriwastav (1975). Studies in Cascade Reliability-I, IEEE Transactions on Reliability , Vol.R-24, No.1, pp.53-57.

[3] A.C.N.Raghava char, B.Kesava Rao and S.N.Narahari Pandit(Sept.1987).The Reliability of a Cascade system with Normal Stress and Strength distribution, ASR, Vol. No.2, pp. 49-54.

[4] T.S.Uma Maheswari (1993). Reliability comparison of an $n$-cascade system with the addition of an $n$ - strength systems, Micro Electron Reliability, Pergamon Press, OXFORD, Vol.33, No.4, pp: 477-479.

[5] T.S.Uma Maheswari(1993). Reliability of cascade system with normal stress and exponential strength, Micro Electron Reliability, Pergamon Press, OXFORD, Vol.33, pp: 927-936.

[6] Rekha, A. and Shyam Sunder, T.( 1997). Reliability of a cascade system with exponential strength and gamma stress, Microelectronics and Reliability, 37, 683-685.

[7] Rekha, A. and Chenchu Raju, V.C.(1999). Cascade system reliability with Rayleigh distribution, Botswana Journal of Technology, $8,14-19$ 